\title{
Glassy Behavior in the Ferromagnetic Ising Model on a Cayley Tree
}

\author{
R. Mélin ${ }^{(1)}$, J.C. Anglès d'Auriac ${ }^{(1)}$, P. Chandra ${ }^{(2)}$ and B. Douçot ${ }^{(1)}$ \\ ${ }^{(1)}$ CRTBT-CNRS, 38042 Grenoble BP 166X cédex France \\ ${ }^{(2)}$ NEC Research Institute, 4 Independence Way, 08540 Princeton NJ, USA
}

\begin{abstract}
We present a detailed study of the nearest-neighbor ferromagnetic Ising model on a Cayley tree. In the limit of zero field, the system displays glassy behavior below a crossover temperature, $T_{g}$, that scales inversely with the logarithm of the number of generations; thus $T_{g}$ is inversely proportional to the logarithm of the logarithm of the number of sites. Non-Gaussian magnetization distributions are observed for $T<T_{g}$, reminiscent of that associated with the central spin of the Edwards-Anderson model on the same tree; furthermore a dynamical study indicates metastability, long relaxation times and ageing consistent with the development of glassy behavior for a finite but macroscopic number of sites.
\end{abstract}




\section{Introduction}

Recursive structures like the Bethe lattice and the Cayley tree provide a pedagogical enviroment for the study of physical problems; in this setting they can be treated with a direct analytic approach without resorting to approximate methods. [1] The Bethe lattice, an infinite Cayley tree, is a connected dendritic structure with constant coordination, $z$, and no loops, as displayed for $z=3$ in Figure 1 . Strictly speaking it is a pseudo-lattice since it cannot be embedded in any real finite-dimensional lattice; indeed it is often regarded as an infinite-dimensional structure since the number of sites accessible in $N$ steps from a given site $\left(\sim N^{d}\right.$ for a $d$-dimensional lattice $)$ increases exponentially with $N$. Thus the Bethe lattice provides a setting where mean-field treatments can become exact. This property was first discussed by Domb who showed that the Bethe-Peierls (BP) approximation to the nearest-neighbor (nn) ferromagnetic (FM) Ising problem, with $H=-J \sum_{(i j)} \sigma_{i} \sigma_{j}$ where $J>0, \sigma_{i}= \pm 1$ and $(i j)$ indicates a $\mathrm{nn}$ sum, is exact on this structure; [2] its solution is identical to that of the infinite-range FM Ising model. [3] Similarly Thouless, Anderson and Palmer studied the infinite-range Sherrington-Kirkpatrick (SK) model of spin glasses on the Bethe lattice using a mean-field technique; [5] they were able to recover the key results[6] of Sherrington and Kirkpatrick (SK) without using the replica method. More recently there have been several studies of the SK model on the Bethe lattice, particularly in finite fields.[0, 8, 9, 10, 11, 12, 13, 14, 15] In general the study of a variety of problems on this recursive structure has helped to develop our understanding of diverse physical phenomena including self-avoiding polymers, 16] random resistor networks 17] and percolation. [18]

Like the Bethe lattice, a Cayley tree is a connected structure with fixed coordination number and no loops; however it has a finite number of generations (cf. Figure 1) and hence sites that are dominated by the boundary. More specifically, the total number of sites in a Cayley tree of $n$ generations with coordination $z$ is

$$
N=1+z+z(z-1)+\ldots z(z-1)^{n-1}=\frac{\left(z(z-1)^{n}-2\right)}{(z-2)}
$$

and the number of surface atoms is

$$
N_{s}=z(z-1)^{n-1}
$$

so that for large $n$

$$
\frac{N_{s}}{N} \approx \frac{(z-2)}{(z-1)}
$$

in contrast to the situation in "real" lattices $\left(\frac{N_{s}}{N} \sim N^{-\frac{1}{d}}\right)$. Thus the "interior" of a Cayley tree, in the limit of a large number of generations, contains an arbitrarily small fraction of its total number of sites, and the boundary plays a key role in any problem studied on this graph. In particular, the BethePeierls transition for the FM Ising model on a Cayley tree occurs only for its central spin; despite its finite moment, the total spontaneous magnetization of all the spins remains zero. [19, 20, 21, 22] In a 
nutshell this occurs because, at zero field and low temperatures, very large domains of flipped spins can nucleate from the boundaries; the resulting finite-size glassiness is the subject of this Paper.

The recursive structure of the Cayley tree permits a detailed analysis of the single-site magnetization distribution as a function of field and generation. In doing so, we find that for fields $h<h_{c 0}$, where the crossover field $h_{c 0}$ decreases exponentially with the number of generations, there is a temperature-scale $T_{g}$ below which well-defined, large domains of flipped spins exist. For $T<T_{g}$ the magnetization distribution becomes non-Gaussian, reminiscent of that associated with the central spin of the magnetized spin glass phase of the $\pm J$ model on the Cayley tree. The cross-over temperature, $T_{g}$, scales inversely with the logarithm of the number of generations of the Cayley tree so that the "finite-size" glassiness persists to very large system sizes for a macroscopic number of sites.

We therefore have a short-ranged periodic spin model that has a "glass" cross-over temperature that decreases very slowly with increasing system size; more specifically it is inversely proportional to the logarithm of the logarithm of the number of sites. We characterize it using a combination of analytic and numerical techniques, always retaining open boundary conditions. First we study the magnetization for different thermodynamic limits emphasizing the crucial role of the ratio of surface/bulk sites as $n \rightarrow \infty$. We recover the Bethe-Peierls result if this ratio goes to zero; otherwise, for vanishing applied fields, there is a cross-over to a glassy phase characterized by well-defined clusters of flipped spins. We find in that for $h<h_{c o}$, the single-site magnetization distribution becomes non-Gaussian for $T<T_{g}$ similar to that of spin glass models residing on the same structure; however it recovers its Gaussian character with increasing field. For $T<T_{g}$ the largest barriers associated with developing broken bonds in these domains scale with the number of cluster sites; we thus refer to this low-temperature state as a finite-size glass. A dynamical study of this system, performed numerically, indicates the presence of metastable states and long relaxation times at low

temperatures. The autocorrelations for $T<T_{g}$ are determined after a waiting time, and indicate ageing effects; the variation of $\chi_{1}^{\prime}$ and $\chi_{3}^{\prime}$ with temperature also agree with the presence of glassiness. As expected, the Edwards-Anderson susceptibility of the entire tree has a maximum which develops slowly with system-size; no divergence is observed. Finally we find that for this system the density of Lee-Yang zeroes in the presence of a complex field is very high in the vicinity of the real axis below a particular temperature-scale; we identify it as the crossover temperature $T_{g}$. We end with a summary of our results and plans for future work.

\section{The Different Thermodynamic Limits on the Cayley Tree}

\subsection{Warm-Up: The Bethe-Peierls Transition of the Central Spin}

One way to take the thermodynamic limit on an $n$-generation Cayley tree is to look solely at the properties of its central spin, and then to take the limit $n \rightarrow+\infty$. As first pointed out in [4], the 
behavior of the central spin is then characteristic of an infinite-dimensional lattice; more specifically it displays a mean-field transition. In other words, the Bethe-Peierls approximation becomes exact on a finite Cayley tree if and only if one considers solely the properties of its central spin and ignores its surface. For recent results in this field, we refer the reader to reference [16] In appendix A we give the calculation of the recursion relation for the partition function $Z_{n}\left(\beta, H, H_{n}\right)$ of an $n$-generation tree with coordination $z$; here $\beta$ is the inverse temperature and $H$ and $H_{n}$ are the magnetic fields acting on the spins of generations 0 to $n-1$ and $n$ respectively. The resulting recursion relation for $Z_{n}\left(\beta, H, H_{n}\right)$ is

$$
Z_{n}\left(\beta, h, h_{n}\right)=\left(4 \cosh ^{2}(\beta J) \cosh ^{2}\left(\beta h_{n}\right)\right)^{\frac{(z-1)^{n}}{2}} Z_{n-1}\left(\beta, h, h+T . h_{n}\right),
$$

where the transformation of the magnetic field is

$$
T . h=\frac{1}{2 \beta} \ln \frac{\cosh \beta(J+h)}{\cosh \beta(J-h)} .
$$

We consider the special case of a field $\epsilon$ applied at the surface of a tree, and ask whether it is amplified in the bulk as determined by the recursion relation (5). This condition defines the bulk critical temperature $\beta_{c}$ by the expression $(z-1) \tanh \beta_{c} J=1$. If $\beta<\beta_{c}$, the magnetization of the central spin is zero; however if $\beta>\beta_{c}$, there is a broken symmetry for the central spin in the thermodynamic limit. As discussed in [3], the critical behavior of the central spin in the thermodynamic limit is identical to that of an infinite-range ferromagnet where $\beta=1 / 2$ and $\delta=3$.

\subsection{Beyond the Bethe Peierls Regime: Different Thermodynamic Limits}

We now wish to look at the transition, not only of the central spin, but of the entire tree. First we consider only half-space-trees, that is trees such that the coordination associated with the ancestor is $z-1$ and not $z$. In what follows, we denote a half-space-tree of $n$ generations as a $n$-half-space-tree; one is pictured on figure 2. We shall label the generations so that the ancestor is at generation $n$ and the leaves are at generation 1 . We are interested in the magnetic properties of the spins in generations $n-m$ to $n$ in the limit $n \rightarrow+\infty$ where, of course, $m$ is a function of $n$; more specifically we want to classify the different regimes as a function of $m(n)$ in an external uniform field $h$. In order to obtain the magnetization of the generations $n-m$ to $n$, we must apply a source magnetic field $\lambda$ to these generations; then we differentiate the partition function with respect to $\lambda$ in the limit $\lambda \rightarrow 0$ to obtain

$$
\langle M(n, m, h)\rangle=\frac{\partial}{\partial(\beta \lambda)} \ln Z(n, m, h, \lambda=0) .
$$

Details of the calculation of the partition function are given in appendix B, and we obtain

$$
\langle M(n, m, h)\rangle=\sum_{i=n-m}^{n-1}(z-1)^{n-i} \frac{\sinh \beta h_{i} \cosh \beta h_{i}}{\cosh ^{2} \beta J+\sinh ^{2} \beta h_{i}} \frac{d h_{i}}{d \lambda}(\lambda=0)+\tanh \beta h_{n} \frac{d h_{n}}{d \lambda}(\lambda=0) .
$$


The last term is the contribution of the ancestor to the average magnetization; $h_{i}$ is the total field at generation $i$ which is the sum of the external, the source and the recursive (of (5) fields ( $h, \lambda$ and $h^{\text {ind }}$ ) respectively.

We do not treat the iteration of $h^{\text {ind }}$ exactly, but approximate it as described below. From the iteration (5) we can deduce the shape of $h_{i+1}^{i n d}$ as a function of $h_{i}$, which is plotted on figure 3. If $\beta<\beta_{c}$, the slope at the origin is less than unity, whereas it is larger than unity for $\beta>\beta_{c}$. Moreover for $\beta>\beta_{c}$ there is one non-trivial fixed point, for which $h_{i+1}^{i n d}=h_{i}=h^{*}$ which depends only on the temperature. This behavior suggests that the iteration can be approximated by linearizing $h_{i+1}\left(h_{i}\right)$ in the vicinity of $h_{i}=0$ and of $h_{i}=h^{*}$. More precisely, we deduce from (5) that

$$
\frac{d h_{i+1}^{i n d}}{d h_{n}}=(z-1) \frac{\sinh \beta J \cosh \beta J}{\cosh ^{2} \beta J+\sinh ^{2} \beta h_{i}} .
$$

and define new variables, $\eta_{1}$ and $\eta_{2}$, such that

$$
\begin{aligned}
\frac{d h_{i+1}^{i n d}}{d h_{i}}\left(h_{i}=0\right) & =(z-1) \tanh \beta J \equiv 1+\eta_{1} \\
\frac{d h_{i+1}^{i n d}}{d h_{i}}\left(h_{i}=h^{*}\right) & \equiv 1-\eta_{2},
\end{aligned}
$$

where $\eta_{1} \in[0, z-2]$ and $\eta_{2} \in[0,1]$ and we note that $\eta_{1}$ and $\eta_{2}$ depend only on the temperature. We then express the complete recursion by

$$
h_{i+1}^{i n d}=\left(1+\eta_{1}\right) h_{i}
$$

or

$$
h_{i+1}^{i n d}=\eta_{2} h^{*}+\left(1-\eta_{2}\right) h_{i}
$$

The first linearization corresponds to $h_{i} \in\left[0, h_{c}\right]$ and the second one to $h_{i} \in\left[h_{c}, h^{*}\right]$, where

$$
h_{c}=\frac{\eta_{2}}{\eta_{1}+\eta_{2}} h^{*} \text {. }
$$

If $\lambda=0$, iteration of the total magnetic field $h_{n}$ leads to different results depending on the relative magnitude of $i$ (generation) compared with

$$
n_{c}(h)=\left[\frac{1}{\ln \left(1+\eta_{1}\right)} \ln \left(\frac{\eta_{1} \eta_{2}}{\eta_{1}+\eta_{2}} \frac{h^{*}}{h}+1\right)\right]
$$

where [] denotes the integer part; for $i \leq n_{c}(h)$

$$
h_{i}=\frac{h}{\eta_{1}}\left(\left(1+\eta_{1}\right)^{i+1}-1\right)
$$

and if $i \geq n_{c}(h)$.

$$
h_{i}=h^{*}+\frac{h}{\eta_{2}}+\left(h_{n_{c}}-h^{*}-\frac{h}{\eta_{2}}\right)\left(1-\eta_{2}\right)^{i-n_{c}} .
$$

There are therefore three regimes which we can study corresponding to the application of large (I), intermediate (II) and vanishing (III) fields on the finite Cayley tree; more specifically they correspondand to the conditions $0 \leq n_{c} \leq n-m$ (I), $n-m+1 \leq n_{c} \leq n$ (II) and $n_{c} \geq n$ (III) respectively where $n_{c}$ is defined in equation (14). 


\subsubsection{Regime I: $0 \leq n_{c} \leq n-m$}

We now determine the average magnetization as given by equation (7) in Regime I. Using the approximation discussed in the previous section, we write

$$
\frac{d h_{i}}{d \lambda}(\lambda=0)=\frac{1}{\eta_{2}}\left(1-\left(1-\eta_{2}\right)^{i-n+m+1}\right) .
$$

If $0 \leq h_{i} \leq h_{c}$ or $i<n_{c}$, we can approximate

$$
\frac{\sinh \beta h_{i} \cosh \beta h_{i}}{\cosh ^{2} \beta J+\sinh ^{2} \beta h_{i}} \simeq a_{1} h_{i}
$$

where

$$
a_{1}=\frac{\beta}{\cosh ^{2} \beta J}
$$

and if $h_{i} \geq h_{c}$ or $n \geq n_{c}$, we use

$$
\frac{\sinh \beta h_{i} \cosh \beta h_{i}}{\cosh ^{2} \beta J+\sinh ^{2} \beta h_{i}} \simeq a_{2}\left(h_{i}-h^{*}\right)+b_{2},
$$

where

$$
\begin{aligned}
a_{2} & =\frac{\cosh ^{2} \beta J \cosh ^{2} \beta h^{*}+\sinh ^{2} \beta J \sinh ^{2} \beta h^{*}}{\left(\cosh ^{2} \beta J+\sinh ^{2} \beta h^{*}\right)^{2}} \\
b_{2} & =\beta \frac{\sinh \beta h^{*} \cosh \beta h^{*}}{\cosh ^{2} \beta J+\sinh ^{2} \beta h^{*}} .
\end{aligned}
$$

It is now straightforward to insert these expressions into the equation (7) for the average magnetization. Since we normalize by the number of sites

$$
N_{m}=1+(z-1)+\ldots+(z-1)^{m}=\frac{(z-1)^{m+1}-1}{z-2},
$$

it is reasonable to neglect the contribution of the ancestor. We obtain

$$
\begin{aligned}
& \langle M(n, m, h)\rangle=\frac{1}{\eta_{2}}\left[\left(b_{2}+a_{2} \frac{h}{\eta_{2}}\right) N_{m}\right. \\
+ & (z-1)^{m} \frac{1-\left(\left(1-\eta_{2}\right) /(z-1)\right)^{m}}{1-\left(\left(1-\eta_{2}\right) /(z-1)\right)}\left(-\left(b_{2}+a_{2} \frac{h}{\eta_{2}}\right)\left(1-\eta_{2}\right)+a_{2}\left(h_{n_{c}}-h_{\infty}\right)\left(1-\eta_{2}\right)^{n-m-n_{c}}\right) \\
- & \left.a_{2}\left(h_{n_{c}}-h_{\infty}\right)(z-1)^{m}\left(1-\eta_{2}\right)^{n-m-n_{c}+1} \frac{1-\left(\left(1-\eta_{2}\right)^{2} /(z-1)\right)^{m}}{1-\left(\left(1-\eta_{2}\right)^{2} /(z-1)\right)}\right],
\end{aligned}
$$

where $h_{\infty}=h^{*}+h / \eta_{2}$. We can now discuss the different thermodynamic limits. First we note that if $n \rightarrow \infty, m \rightarrow \infty$ and $n-m \rightarrow \infty$, the thermodynamic limit of the normalized magnetization reads

$$
\lim \frac{\langle M(n, m, h)\rangle}{N_{m}}=\left(b_{2}+a_{2} \frac{h}{\eta_{2}}\right) \frac{z-1}{z-2+\eta_{2}} .
$$


However if we take a different thermodynamic limit, with $n-m \rightarrow b$, where $b$ is a constant thickness boundary, we obtain

$$
\lim \frac{\langle M(n, m, h)\rangle}{N_{m}}=\left(b_{2}+a_{2} \frac{h}{\eta_{2}}\right) \frac{z-1}{z-2+\eta_{2}}-a_{2}\left(h_{\infty}-h_{n_{c}}\right) \frac{(z-1)(z-2)\left(1-\eta_{2}\right)^{b-n_{c}}}{\left(z-2+\eta_{2}\right)\left(z-1-\left(1-\eta_{2}\right)^{2}\right)}
$$

The absolute value of the corrective term decreases as the thickness of the boundary increases since $\left|1-\eta_{2}\right|<1$.

\subsubsection{Regime II: $n-m+1 \leq n_{c} \leq n$}

Again we calculate the average magnetization, (]), this time in the regime $n-m+1 \leq n_{c} \leq n$ where we use the relations (18) and (20) from the previous section. Furthermore we need to approximate the expression $d h_{i} / d \lambda$ for $\lambda=0$. If $n-m \leq i \leq n_{c}$, we have

$$
\frac{d h_{i}}{d \lambda}(\lambda=0)=\frac{1}{\eta_{1}}\left(\left(1+\eta_{1}\right)^{i-n+m+1}-1\right),
$$

and if $i \geq n_{c}$,

$$
\frac{d h_{i}}{d \lambda}(\lambda=0)=\frac{1}{\eta_{2}}+a\left(1-\eta_{2}\right)^{i-n_{c}}
$$

where

$$
a=\frac{1}{\eta_{1}}\left(1+\eta_{1}\right)^{n_{c}-n+m+1}-\frac{1}{\eta_{1}}-\frac{1}{\eta_{2}} .
$$

We have used

$$
h_{i}=h^{*}+\frac{h+\lambda}{\eta_{2}}+\left(1-\eta_{2}\right)^{i-n_{c}}\left(h_{n_{c}}(\lambda)-h^{*}-\frac{h+\lambda}{\eta_{2}}\right)
$$

with

$$
h_{n_{c}}(\lambda)=h_{n_{c}}(0)+\frac{\lambda}{\eta_{1}}\left(\left(1+\eta_{1}\right)^{n_{c}-n+m+1}-1\right) .
$$

We insert these expressions into (đ) and sum the full geometric series. We note that the thermodynamic limit can only be taken with $n-m \rightarrow b$, where again $b$ is a constant. We find that the dominant behavior at small magnetic field $h$ depends on the temperature. Let $T^{\prime}$ be the temperature such as $\tanh \beta^{\prime}=1 / \sqrt{z-1}$. Then if $T^{\prime}<T<T_{c}$ the dominant term in the normalized magnetization is linear in $h$ with corrections in $h^{\alpha}$, where $\alpha=\ln (z-1) / \ln \left(1+\eta_{1}\right)-1$; by contrast if $T<T^{\prime}$ the leading term is of order $h^{\alpha}$. We do not write explicitly the corresponding expressions since they are tedious and do not contribute further to the present discussion.

\subsubsection{Regime III: $n_{c} \geq n$}

In this regime, we approximate the average magnetization by inserting (18) and (27) into (7) which yields

$$
\langle M(n, m, h)\rangle=\sum_{i=n-m}^{n-1}(z-1)^{n-i} a_{1} h \frac{\left(1+\eta_{1}\right)^{i+1}-1}{\eta_{1}} \frac{\left(1+\eta_{1}\right)^{i-n+m+1}-1}{\eta_{1}} .
$$


After summing the geometrical series, we obtain

$$
\begin{aligned}
\frac{\langle M(n, m, h)\rangle}{N(m)}= & \frac{a_{1} h}{\eta_{1}^{2}} \frac{z-2}{1-(z-1)^{-m-1}}\left[\frac{1-(z-1)^{-m}}{z-2}-\frac{\left(1+\eta_{1}\right)\left(\left(1+\eta_{1}\right)^{b}+1\right)}{z-2-\eta_{1}}\left(1-\left(\frac{1+\eta_{1}}{z-1}\right)^{m}\right)\right. \\
& \left.+\frac{\left(1+\eta_{1}\right)^{b+2}}{z-1-\left(1+\eta_{1}\right)^{2}}\left(1-\left(\frac{\left(1+\eta_{1}\right)^{2}}{z-1}\right)^{m}\right)\right]
\end{aligned}
$$

We identify the cross-over field $h_{c o}$ as $h_{n-1}=h_{c}$, that is

$$
h_{c o}=h^{*} \frac{\eta_{1} \eta_{2}}{\left(\eta_{1}+\eta_{2}\right)\left(\left(1+\eta_{1}\right)^{n}-1\right)},
$$

which is exponentially small in $n$. If we take the limit of large $n$ and $m$ but finite $n-m$, we can write

$$
\begin{aligned}
\frac{\left\langle M\left(n, m, h_{c o}\right)\right\rangle}{N(m)}= & h^{*} \frac{a_{1} \eta_{2}}{\eta_{1}\left(\eta_{1}+\eta_{2}\right)}(z-2)\left(1+\eta_{1}\right)^{-n}\left[\frac{1}{z-2}-\frac{\left(1+\eta_{1}\right)\left(\left(1+\eta_{1}\right)^{b}+1\right)}{z-2-\eta_{1}}\left(1-\left(\frac{1+\eta_{1}}{z-1}\right)^{m}\right)\right. \\
& \left.+\frac{\left(1+\eta_{1}\right)^{b+2}}{z-1-\left(1+\eta_{1}\right)^{2}}\left(1-\left(\frac{\left(1+\eta_{1}\right)^{2}}{z-1}\right)^{m}\right)\right]
\end{aligned}
$$

In the limit $T \rightarrow 0,\left(1+\eta_{1}\right) /(z-1)=\tanh \beta \rightarrow 1$, so that the only non-negligible term is

$$
\frac{\left(1+\eta_{1}\right)^{-n}\left(1+\eta_{1}\right)^{2 m}}{(z-1)^{m}}=\left(1+\eta_{1}\right)^{-b}\left(\frac{1+\eta_{1}}{z-1}\right)^{m} .
$$

Since

$$
\ln \left(\frac{1+\eta_{1}}{z-1}\right)^{m} \simeq-2 m e^{-2 \beta}
$$

at small temperature, we obtain the cross-over temperature scale $T_{g}=2 / \ln m$. In the next section we shall rederive this crossover temperature with another method and give its significance.

\section{Magnetization Distribution and Finite-Size Effects}

In this section we study the distribution of the magnetization which can be determined from exact recursion relations. We find that finite-size effects are crucial in this analysis, and we recover the crossover temperature $T_{g}$ discussed in the previous section. We also compute the distribution of the magnetization in finite field.

\subsection{Magnetization distribution in zero field}

We begin with the case of zero magnetic field. Given $z-1 n$-half-space trees with coordination $z$, it is straighforward to obtain a $(n+1)$-half space tree with the same coordination. One just has to add a common ancestor and to link it ancestor to the $z-1$ ancestors of each $n$-tree (see figure 2). In order to get a full tree, one has to "glue" $z$ half-space-trees instead of $z-1$ at the last step. Let 
$P_{n}^{\sigma}(M)$ be the conditional probability for a $n$-half-space-tree to have a magnetization $M$, given the spin of the ancestor is $\sigma$. Of course,

$$
\sum_{M} P_{n}^{\sigma}(M)=1
$$

The recursion relation for $P_{n}^{\sigma}(M)$ is

$$
\begin{aligned}
P_{n}^{\sigma}(M)= & \sum_{M_{1}, \ldots, M_{z-1}} \delta\left(M-\left(M_{1}+\ldots+M_{z-1}+\sigma\right)\right) \\
& \sum_{k=0}^{z-1}\left(\begin{array}{c}
z-1 \\
k
\end{array}\right) x^{k}(1-x)^{z-k-1} \prod_{i=1}^{k} P_{n-1}^{-\sigma}\left(M_{i}\right) \prod_{i=k+1}^{z-1} P_{n-1}^{\sigma}\left(M_{i}\right),
\end{aligned}
$$

where $x$ is the probability for breaking one bond:

$$
x=\frac{e^{-\beta J}}{e^{\beta J}+e^{-\beta J}} .
$$

The initialization of the recursion is given by $P_{1}^{\sigma}\left(\sigma^{\prime}\right)=\delta_{\sigma, \sigma^{\prime}}$. This recursion can be performed numerically, at least for a small number of generations. The result is plotted on figure 1 for $z=3$ and 10 generations. For a finite size tree and at low temperature, the magnetization distribution presents a non gaussian structure, reminiscent of the magnetization distribution of the central spin in Bethe lattice spin glasses [10] [14]. Notice that the temperature which controls the departure from the Gaussian distribution is lower than the bulk transition temperature.

In order to determine precisely this temperature, we compute the recursion relations for the average magnetization. Using the recursion relations (39), we find

$$
\langle M\rangle_{n+1}^{+}=1+\sum_{k=0}^{z-1}\left(\begin{array}{c}
z-1 \\
k
\end{array}\right) x^{k}(1-x)^{z-k-1}\left(k\langle N\rangle_{n}^{-}+(z-k-1)\langle M\rangle_{n}^{+}\right)
$$

It is clear that, since $P_{1}^{+}(\sigma)=P_{1}^{-}(-\sigma)$, for all n $P_{n}^{+}(M)=P_{n}^{-}(-M)$, so that $\langle M\rangle_{n}^{+}+\langle M\rangle_{n}^{-}=0$. Putting this equation into (41) and using the well-known relations for the sum of binomial series, we obtain $\langle M\rangle_{n+1}^{+}=p\langle M\rangle_{n}^{+}+1$ and $\langle M\rangle_{0}^{+}=1$ where $p$ is defined as $p=(z-1) \tanh \beta J$. This recursion can be easily solved, and one gets

$$
\frac{\langle M\rangle_{n}^{+}}{N_{n}}=\frac{z-2}{p-1} \frac{p^{n+1}-1}{(z-1)^{n+1}-1}
$$

where $N_{n}$ is the number of sites of a $n$-half-space-tree, given by (23). The structure of the distribution of magnetization is non-Gaussian provided $2 x n \ll 1$, that is

$$
T<T_{g}=\frac{J}{\ln n}=\frac{J}{\ln \left(\ln N_{n} / \ln (z-1)\right)} .
$$

It is clear that the temperature $T_{g}$ (which will be identified wit the glass temperature) decreases very slowly with the system size. For instance, in limit where $N \simeq 6.02 .10^{23}$ and $z=3, T_{g}=J / 4.4$. We 
conclude from this analysis that finite-size effects persist in the limit of a macroscopic number of sites. For $T_{g}$ to be drastically reduced, one should consider systems of size exp 6.02.10 ${ }^{23}$ (!). Since the appearance of glassiness is a finite-size effect, we stress that $T_{g}$ is a cross-over temperature scale even for macroscopic systems.

\subsection{Structure of the Magnetization Distribution for $T<T_{g}$}

We would like to understand qualitatively the structure of the maxima of the magnetization probability distribution below $T_{g}$; more specifically we want localize the maxima and to calculate their weight. To do this, we use the normalized continuous magnetization variable $m=M / N_{n} \in[-1,1]$ and the associated density $\rho_{n}(m)=N_{n} P_{n}^{+}(M)$. The recursion relations for $\rho_{n}(m)$ are derived in a straightforward fashion from those for $P_{n}^{+}(M)$ that are shown in (39). Since this relation is a convolution, we write the recursion in terms of the Fourier transform $\tilde{\rho}_{n}(k)$ of $\rho_{n}(m)$ :

$$
\tilde{\rho}_{n}(k)=\int_{-\infty}^{+\infty} e^{i k m} \rho_{n}(m) d m
$$

We obtain the recursion of the $\tilde{\rho}$ 's:

$$
\begin{aligned}
\tilde{\rho}_{n+1}(k) & =\left(x \tilde{\rho}_{n}\left(-\frac{k}{z-1}\right)+(1-x) \tilde{\rho}\left(\frac{k}{z-1}\right)\right)^{z-1} \\
\tilde{\rho}_{0}(k) & =e^{i k}
\end{aligned}
$$

We now study the special case $z=3$ where the formulae are simpler. It is easy to check by recursion that

$$
\tilde{\rho}_{n}(k)=\left(1-2 x\left(2^{n}-1\right)\right) e^{i k}+2 x \sum_{\alpha=0}^{n-1} 2^{\alpha} \exp \left(i\left(1-\frac{1}{2^{\alpha}}\right) k\right)+O\left(x^{2}\right) .
$$

As we shall see later, this low-temperature expansion is meaningful below $T_{g}$ even in the presence of a finite density of kinks. The expansion (47) tells us that $\rho_{n}(m)$ has peaks for $m_{\alpha}=1-1 / 2^{\alpha}$, where $\alpha \in\langle 0, \ldots, n-1\rangle$. Moreover, we find that the weight of the peak $\alpha+1$ is twice that of $\alpha$. Inspection of figure 1 indicates that this prediction is correct, at least in the region where the overlap between the peaks is small.

These results can also be interpreted in the following fashion. The expansion (47) at order $x$ means that the magnetization density is calculated at the order of one kink. It is clear that a single kink at generation $n-\alpha$ leads to a magnetization $1-1 / 2^{\alpha}$ and that the number of choices to put a kink at generation $n-\alpha-1$ is twice the number of choices to put a kink at generation $n-\alpha$, which is the content of equation (47). What is striking is that below $T_{g}$, this one kink picture is valid, even though we deal with a finite density of kinks $x$. This means that $T_{g}$ is the temperature below which the kinks are rarely nested. In order to check this assertion, we determine the condition for when the kinks to "induce" well-defined domains of flipped spins; this criterion will determine the validity of the expansion (47). The overlap between the domains induced by the kinks is small 
provided $x N_{n}\langle S\rangle_{n}<N_{n}$. In this expression, $\langle S\rangle_{n}$ is the average size of a domain of flipped spins induced by a single kink. The number of descendants of a kink at level $n-p$ for a $n$-half-space-tree is $S_{p, n}=1+2+\ldots+2^{n-p}=2^{n-p+1}-1$. The average over $p$ of $S_{p, n}$ is

$$
\left\langle S_{p, n}\right\rangle_{n}=\frac{\sum_{p=1}^{n} 2^{p} S_{p, n}}{\sum_{p=1}^{n} 2^{p}}=n \frac{2^{n}}{2^{n}-1}-1 \simeq n-1 .
$$

The condition for "non-overlap" is $x n<1$ which is just $T<T_{g}$. We conclude that for $T<T_{g}$, the number of kinks is small enough for the system to develop well-defined domains of flipped spins. In this temperature regime, the excitations of the spin system are kinks which are local in the bond variables but are highly non-local in terms of the spins. in terms of bounds, but highly non-local in terms of spins.

\subsection{Magnetization Distribution in a Magnetic Field}

The magnetization distribution in a magnetic field can be computed using

$$
P_{n, h}^{\sigma}(m)=\frac{P_{n, 0}^{\sigma}(m) e^{\beta m h}}{\sum_{m^{\prime}} P_{n, 0}^{\sigma}\left(m^{\prime}\right) e^{\beta h m^{\prime}}} .
$$

The conditional magnetization distribution (the central spin being parallel to the field) is found to converge rapidly to a gaussian as the field increases. Since large domains of flipped spins do not survive in a magnetic field, the cross-over field is expected to decrease drastically with the system size. Indeed, we have shown previously that the cross-over field $h_{c o}$ of equation (34) decreases exponentially with the number of generations. The distribution of magnetizations is plotted on figure 5, where the central spin is taken to be parallel to the field.

\section{Barrier Structure}

We have shown that the excitations below $T_{g}$ are broken bonds. In order to characterize the dynamics, we calculate the barriers associated with these excitations. The energy barriers at zero temperature of a half-space-tree are defined as follows: one starts with a configuration where all the spins are up and then one considers single-spin-flip paths from the initial configuration to a final one where all the spins are reversed. To each such single-spin-flip path, we associate the maximal energy reached during the "passage" from the initial to the final configuration where we take the energy of the former to be zero. Then the barrier is defined as the minimum over all the paths of the maximum energy of one path. Typically, the Monte Carlo algorithm samples all the paths in an ergodic way; by contrast the Swenson algorithm does not generate paths with respect to the single spin flip. In Appendix B we give details of the calculation of the barriers associated with the $n$-half-space-tree. Here we calculate the number of states with a given barrier $E_{\alpha}^{b}$ at a given temperature below $T_{g}$, for a full $n$-tree. Following our previously established convention, the leaves and the center reside at 
the first and the $n$-th generation respectively. We note there exist $n^{*}$ kinks at a given temperature where $n^{*}=N x$. The barrier for a configuration of $n^{*}$ kinks is assumed to be only a function of the generation $\alpha$ of the kink which is the closest to the origin. In order to calculate the number of states with an energy barrier $E_{\alpha}^{b}$, we have to enumerate all the configurations with no kink between generation $\alpha+1$ and $n, n_{\alpha}$ kinks at generation $\alpha$ and $n^{*}-n_{\alpha}$ kinks between generations $\alpha-1$ and 1. We call the number of such configurations of kinks $g(\alpha)$. We make the approximation that the energy barrier of all these configurations is $E_{\alpha}^{b}$ so that its life-time is, according the the Arrhenius law,

$$
\tau_{\alpha}=\tau_{0} \exp \left(\lambda \beta E_{\alpha}^{b}\right)
$$

where $\lambda$ is a constant. Such a configuration of kinks is displayed on figure 6. Clearly, we have

$$
g(\alpha)=\sum_{n_{\alpha}=1}^{n^{*}} g_{\alpha}\left(n_{\alpha}\right)
$$

with

$$
g_{\alpha}\left(n_{\alpha}\right)=\left(\begin{array}{c}
z(z-1)^{n-\alpha-1} \\
n_{\alpha}
\end{array}\right)\left(\begin{array}{c}
\frac{z}{z-2}\left((z-1)^{n-1}-(z-1)^{n-\alpha}\right) \\
n^{*}-n_{\alpha}
\end{array}\right) .
$$

We can calculate the sum and obtain

$$
g(\alpha)=\left(\begin{array}{c}
\frac{z}{z-2}\left((z-1)^{n-1}-(z-1)^{n-\alpha-1}\right) \\
n^{*}
\end{array}\right)-\left(\begin{array}{c}
\frac{z}{z-2}\left((z-1)^{n-1}-(z-1)^{n-\alpha}\right) \\
n^{*}
\end{array}\right),
$$

and we get the probability $P(\alpha)$ for the system to be in a valley with a barrier $E_{\alpha}$ :

$$
P(\alpha)=\left(\begin{array}{c}
N \\
N x
\end{array}\right)^{-1}\left[\left(\begin{array}{c}
N\left(1-(z-1)^{-\alpha}\right) \\
N x
\end{array}\right)-\left(\begin{array}{c}
N\left(1-(z-1)^{-\alpha+1}\right) \\
N x
\end{array}\right)\right],
$$

where we have normalized by the total number of accessible states at a given temperature on a $n$-tree. We have also approximated the number of sites as follows:

$$
N=\frac{z(z-1)^{n-1}-2}{z-2} \simeq \frac{z(z-1)^{n-1}}{z-2} .
$$

Assuming both that $x \ll 1$, and that $(z-1)^{-\alpha} \ll 1$, and using Stirling's formula, we get

$$
P(\alpha) \simeq \exp \left(-\frac{N x}{(z-1)^{\alpha}}\right)-\exp \left(-\frac{N x}{(z-1)^{\alpha-1}}\right) .
$$

If $N z /(z-1)^{\alpha} \ll 1$, we obtain

$$
P(\alpha) \simeq N x \frac{z-2}{(z-1)^{\alpha}}
$$

As expected, $P(\alpha)$ decreases as a function of $\alpha$, which indicates that the long-lived states are less numerous than their short-lived counterparts. 


\section{Glauber Dynamics}

We now study the Glauber dynamics of the spin system on the Cayley tree.

\subsection{The Glauber Matrix}

We begin with a general discussion of Glauber dynamics. Let $p(\{\sigma\}, t)$ be the probability for the system to be in a state $\{\sigma\}$ at time $t$. Following Glauber [23], we define $w_{i}(\{\sigma\})$ as the probability per unit time that the spin $i$ flips from $\sigma_{i}$ to $-\sigma_{i}$, while the others remain fixed. The master equation is

$$
\frac{d}{d t} p(\{\sigma\}, t)=-\left(\sum_{i=1}^{N} w_{i}(\{\sigma\})\right) p(\{\sigma\}, t)+\sum_{i=1}^{N} w_{i}\left(\left\{\sigma_{1}, \ldots,-\sigma_{i}, \ldots, \sigma_{N}\right\}\right) p\left(\left\{\sigma_{1}, \ldots,-\sigma_{i}, \ldots, \sigma_{N}\right\}, t\right) .
$$

Since we require the Boltzman distribution to be a fixed point, the coefficients $w_{i}(\{\sigma\})$ are of the form

$$
w_{i}(\{\sigma\})=\frac{1}{2}\left(1-\sigma_{i} \tanh \left(\beta J \sum_{j \in V(i)} \sigma_{j}\right)\right),
$$

where $V(i)$ is the set of neighbors of the site $i$. If one denotes by $\mathbf{p}(t)$ the $2^{N}$ vector of the $p(\{\sigma\}, t)$, equation (58) can be written as

$$
\frac{d}{d t} \mathbf{p}(t)=\mathbf{G} \cdot \mathbf{p}(t)
$$

where $\mathbf{G}$ is the Glauber matrix. We first show some properties of the matrix $\mathbf{G}$. Since the Boltzman distribution is a steady state of the dynamics, its corresponding eigenvalue is zero whatever the temperature. Even though it is not symmetric, the matrix $\mathbf{G}$ can be diagonalized and its eigenvalues are real; we give the proof of this statement here. The Glauber matrix satisfies detailed balance which means that $\mathbf{G}_{\alpha, \beta} \mathbf{p}_{\beta}^{(0)}=\mathbf{G}_{\beta, \alpha} \mathbf{p}_{\alpha}^{(0)}$ where $\mathbf{p}^{(0)}$ is the Boltzman distribution. As a consequence

$$
\left(\mathbf{p}_{\alpha}^{(0)}\right)^{1 / 2} \mathbf{G}_{\alpha \beta}\left(\mathbf{p}_{\beta}^{(0)}\right)^{1 / 2}=\left(\mathbf{p}_{\beta}^{(0)}\right)^{1 / 2} \mathbf{G}_{\beta \alpha}\left(\mathbf{p}_{\alpha}^{(0)}\right)^{1 / 2}
$$

Let us define a matrix $\mathbf{M}$ such that

$$
\mathbf{M}_{\alpha \beta}=\left(\mathbf{p}_{\alpha}^{(0)}\right)^{1 / 2} \mathbf{G}_{\alpha \beta}\left(\mathbf{p}_{\beta}^{(0)}\right)^{1 / 2}
$$

Then $\mathbf{M}$ is symmetric. Let $\mathbf{p}$ be a right eigenvector of the Glauber matrix. Then

$$
\sum_{\beta} \mathbf{G}_{\alpha \beta} \mathbf{p}_{\beta}=\lambda \mathbf{p}_{\beta}
$$

is equivalent to

$$
\sum_{\beta} \mathbf{M}_{\alpha \beta}\left(\mathbf{p}_{\beta}^{(0)}\right)^{-1 / 2} \mathbf{p}_{\beta}=\lambda\left(\mathbf{p}_{\alpha}^{(0)}\right)^{-1 / 2} \mathbf{p}_{\alpha}
$$


so that $\left(\mathbf{p}_{\alpha}^{(0)}\right)^{-1 / 2} \mathbf{p}_{\alpha}$ is an eigenvector of $\mathbf{M}$. We conclude that $\mathbf{G}$ is diagonalizable, and that all of its eigenvalues are real.

The spectrum in the infinite temperature limit can be understood in the following manner. If we define a state vector $|\psi\rangle$ by

$$
|\psi\rangle=\sum_{\{\sigma\}} f\left((\{\sigma\})\left|\sigma_{1}\right\rangle \otimes \ldots \otimes\left|\sigma_{N}\right\rangle\right.
$$

then its dynamics are

$$
\frac{d}{d t}|\psi\rangle=-\frac{N}{2}|\psi\rangle+\frac{1}{2} \sum_{i=1}^{N} \sigma_{i}^{x}|\psi\rangle
$$

so that the eigenvalues of the Glauber matrix at infinite temperature are of the form

$$
\lambda=-\frac{N}{2}+\frac{1}{2} \sum_{i=1}^{N} \mu_{i}
$$

where $\mu_{i}= \pm 1$. The spectrum in the infinite temperature limit is composed of levels at integer values between $-N$ and 0 , with a degeneracy given by the binomial coefficients.

For bipartite lattices, such as the square lattice of the Caley tree, the spectrum of the matrix $\mathbf{G}$ is symmetric; more specifically if $\lambda$ belongs to the spectrum, then $-N-\lambda$ is an eigenvalue too. We give the proof of this statement now. Let $\mathbf{X}\{\sigma\}$ be an eigenvector of $\mathbf{M}$, with an eigenvalue $\lambda$ :

$$
\lambda \mathbf{X}\{\sigma\}=-\sum_{i=1}^{N} \frac{1}{2}\left(1-\sigma_{i} \tanh \left(\beta J h_{i}\right) \mathbf{X}\{\sigma\}+\sum_{i=1}^{N} \frac{1}{2 \cosh \beta J h_{i}} \mathbf{X}\left\{\sigma_{1}, \ldots,-\sigma_{i}, \ldots, \sigma_{N}\right\},\right.
$$

where $h_{i}$ is defined by

$$
h_{i}=\sum_{j \in V(i)} \sigma_{j}
$$

Let $\mathbf{Y}\{\sigma\}$ be defined as

$$
\mathbf{Y}\{\sigma\}=(-1)^{\nu\{\sigma\}} \mathbf{X}\{\tilde{\sigma}\}
$$

where $\nu\{\sigma\}$ is the number of up spins in the configuration $\{\sigma\} .\{\tilde{\sigma}\}$ is deduced from $\{\sigma\}$ by flipping the spins of one of the two sublattices. Then,

$$
\begin{aligned}
(\mathbf{M Y})\{\sigma\} & =-\sum_{i=1}^{N} \frac{1}{2}\left(1-\sigma_{i} \tanh \left(\beta H h_{i}\right)\right)(-1)^{\nu\{\sigma\}} \mathbf{X}\{\tilde{\sigma}\}+\sum_{i=1}^{N} \frac{(-1)^{\nu\left\{\sigma_{1}, \ldots,-\sigma_{i}, \ldots, \sigma_{N}\right\}}}{2 \cosh \left(\beta J h_{i}\right)} \mathbf{X}\left\{\tilde{\sigma}_{1}, \ldots,-\tilde{\sigma}_{i}, \ldots, \tilde{\sigma}_{M}\right\} \\
& =(-1)^{\nu\{\sigma\}}\left[-\sum_{i=1}^{N} \frac{1}{2}\left(1+\tilde{\sigma}_{i} \tanh \left(\beta J h_{i}\right)\right) \mathbf{X}\{\tilde{\sigma}\}-\sum_{i=1}^{N} \frac{1}{2 \cosh \left(\beta J h_{i}\right)} \mathbf{X}\left\{\tilde{\sigma}_{1}, \ldots,-\tilde{\sigma}_{i}, \ldots, \tilde{\sigma}_{N}\right\}\right] \\
& =-(N+\lambda)(-1)^{\nu\{\sigma\}} \mathbf{X}\{\tilde{\sigma}\}=-(N+\lambda) \mathbf{Y}\{\sigma\} .
\end{aligned}
$$

Given an eigenvector $\mathbf{X}$ for the eigenvalue $\lambda$, we have constructedd an eigenvector $\mathbf{Y}$ for the eigenvalue $-N-\lambda$. 
The difference between (60) and the Schrödinger equation is that quantum mechanics preserves the scalar product which results in Hermitian Hamiltonians. Furthermore, the physical states "reside" in a Hilbert space, and each state of this Hilbert space is physical. In the case of the Glauber matrix there is no such vectorial space; more specifically, the sum of two probability distributions is not a probability distribution. However some quantities are conserved by the dynamics. It is easy to show that the eigenvectors of $\mathbf{G}$ for the non-zero eigenvalues have the property that

$$
\sum_{\{\sigma\}} \mathbf{p}\{\sigma\}=0
$$

This is a simple consequence of the fact that the Glauber matrix preserves the quantity

$$
\sum_{\{\sigma\}} \mathbf{p}\{\sigma\}
$$

\subsection{Spectrum of the Glauber Matrix}

We now discuss the spectrum of the Glauber dynamics as a function of temperature. We have performed a series of numerical diagonalizations on small clusters, and then speculate on the behavior for larger systems specifically with respect to the statistics of their eigenvalues. Since this aspect of the Glauber dynamics has not studied in much detail before, we examine the Ising model on oneand two- dimensional lattices with this approach before ending with the Cayley tree; in particular, we examine their relaxation spectra as a function of temperature and attempt to extrapolate their behavior in the thermodynamic limit.

We begin with the Ising chain, where we have determined the spectrum as a function of temperature for a six-site system with open boundary conditions; the result is displayed in figure 7 . We also treated the case of a 10 sites ring. Of course, we did not follow the 1024 levels as a function of temperature, but computed the eigenvalues for a given temperature. The spectrum is plotted on figure 8. From these results, we can speculate on its behavior for large systems. The spectrum is completly integrable in the sense that no level repulsion occurs in the evolution of the eigenvalues as a function of temperature. It is clear from figure 7 that the evolution of $1 / \tau_{i}$ is monotonous as a function of $\beta$ and that no repulsion is observed. However, the distribution of eigenvalues is non uniform, since a curvature can be seen on figure 8. It is not surprising that the spectrum reveals an underlying integrable dynamics since the dynamics was indeed integrated by Glauber in [23]. We also note the existence of a gap at non-zero temperature, which vanishes in the limit of zero temperature; here the eigenvalue is doubly degenerate, corresponding to the existence of a symmetry-breaking phase at $T=0$.

We have also studied small clusters (of size 3x3) of the two-dimensional Ising model; the resulting spectrum in the low-temperature phase is plotted on figure 9. The obvious difference with the results for the one-dimensional chain is the presence of clusters of relaxation times in the low-temperature 
spectrum. The evolution of the eigenvalues as a function of the inverse temperature is plotted for a small $3 \times 2$ cluster on figure 10. Level repulsion is visible in the vicinity of the transition temperature. For an infinite size system, one expects the presence of a gap between zero and the first negative eigenvalue in the spectrum for $T>T_{c}$; it should vanish at the transition temperature, leading to a doubly-degenerate zero eigenvalue in the interval $[0, T c]$ which corresponds to the existence of a symmetry-breaking stable phase. Thinking of the spectrum as a function of $\beta$, one expects the spectrum to display chaotic behavior in the vicinity of the transition temperature, leading to a rearrangement of the inverse relaxation times at low temperature and the presence of clusters of relaxation times in the zero-temperature limit. However, it is not clear whether the level-spacing statistics should be Gaussian Orthogonal Ensemble (G.O.E.) in character in an appropriate sector; one might be left with a mixture of G.O.E. and Poisson statistics. A better understanding of these level-spacing statistics would involve the search of the symetry sectors using group theory which will be pursued in a future project.

In our study of the Cayley tree, we determined the spectrum of eigenvalues as a function of temperature for $z=3$ nad $n=1$, which corresponds to four sites. The resulting "spaghetti" are plotted in figure 11. Though the system size is very small, one can still see repulsion of the eigenvalues in the vicinity of the bulk transition temperature. We determined the spectrum of the Glauber matrix at low temperatures for $n=2$, resulting in 1024 eigenmodes; the eigenvalues are plotted on figure 12 . The spectrum displays clusters but, unlike the case of the two-dimensional Ising model, these clusters are equidistant and occur at integer values. Furthermore the $\lambda=0$ cluster contains 8 eigenvalues whereas it contained just two for the 2D Ising model. For the case of $N=10$ and $\beta=2$, shown in figure 12, we find that $n^{*} \simeq 0.18<1$. In the previous section, we conclude from equation(57) that $P(\alpha)$ is independent of the temperature; this result is confirmed by the spectrum of the Glauber matrix since the $\lambda=0$ and the $\lambda=-1$ clusters are separated by a gap.

\subsection{Determination of the Glauber Dynamics}

We now diagonalize the Glauber matrix in order to determine the dynamics of the model in an explicit fashion. Let $\mathbf{u}_{\alpha}$ be the eigenvectors of $\mathbf{G}$ : $\mathbf{G} \cdot \mathbf{u}_{\alpha}=\lambda_{\alpha} \mathbf{u}_{\alpha}$, and let $\mathbf{P}$ be the passage matrix from the natural basis of pure states $\mathbf{e}_{\alpha}$ to the basis $\mathbf{u}_{\alpha}$ :

$$
\mathbf{u}_{\alpha}=\sum_{\beta} \mathbf{P}_{\alpha \beta} \mathbf{e}_{\beta}
$$

We look for the temporal evolution of the states $\mathbf{e}_{\alpha}$. At the initial time $(\mathrm{t}=0)$

$$
\mathbf{p}_{\alpha}(0)=\mathbf{e}_{\alpha}=\sum_{\beta} \mathbf{P}_{\alpha \beta}^{-1} \mathbf{u}_{\beta}
$$

and at $t>0$, the state is a mixture of pure states and is given by

$$
\mathbf{p}_{\alpha}(t)=\sum_{\beta} \mathbf{P}_{\alpha \beta}^{-1} e^{\lambda_{\beta} t} \sum_{\gamma} \mathbf{P}_{\beta \gamma} \mathbf{e}_{\gamma}
$$


We can easily compute the magnetization of $\mathbf{p}_{\alpha}(t)$. We applied this procedure to the case of a tree with one generation. The evolution of the magnetization of the 16 pure states is plotted on figure 13 at low temperature. Of course, on very long time scales, the magnetization of all the states relaxes to zero, due to the fact that the eigenvalues associated to the symmetry breaking state are not strictly zero. The evolution of the pure states indicate the existence of metastable states, which are the precursors of the metastable states present for larger values of the number of generations.

\subsection{The Factorization Approximation}

We recover the bulk critical temperature by considering the asymptotics of a simplified Glauber dynamics. Glauber has shown [23] that one can replace the $2^{N}$ variables of the linear dynamics by a hierarchy of $N$, nonlinear, coupled equations for the correlations functions. This procedure is very similar to the transformation of the Liouville equation into the B.B.G.K.Y. hierarchy in the kinetic theory. The first equation of the hierarchy is

$$
\frac{d}{d t} q_{i}(t)=-q_{i}(t)+\left\langle\tanh \left(\beta J \sum_{j \in V(i)} \sigma_{j}\right)\right\rangle .
$$

In this expression, $q=\langle\sigma\rangle$ and $V(i)$ is the set of neighbors of site $i$. In the case of a one dimensional chain, one can use the fact that

$$
\left\langle\tanh \beta J\left(\sigma_{i-1}+\sigma_{i+1}\right)\right\rangle=\frac{1}{2} \tanh (\beta J)\left(q_{i-1}+q_{i+1}\right),
$$

and one gets a closed equation for the 1-point correlation functions. It is also clear that the whole hierarchy decouples, and that one can use this decoupling to integrate the dynamics. In the case of a $z=3$ tree, one has to take into account the fact that the sites inside the tree have three neighbors; by contrast the leaves have one neighbor. For this coordination,

$$
\tanh \beta J\left(\sigma_{1}+\sigma_{2}+\sigma_{3}\right)=\alpha\left(\sigma_{1}+\sigma_{2}+\sigma_{3}\right)+\gamma\left(\sigma_{1}+\sigma_{2}+\sigma_{3}\right)^{3}
$$

where the coefficients $\alpha$ and $\gamma$ are determined by

$$
\begin{aligned}
\alpha & =\frac{1}{24}(27 \tanh \beta J-\tanh 3 \beta J) \\
\gamma & =\frac{1}{24}(\tanh 3 \beta J-3 \tanh \beta J) .
\end{aligned}
$$

We can thus obtain the first equation of the hierarchy in the case of the $z=3$ tree. For the sites with three neighbours,

$$
\frac{d}{d t} q_{i}=-q_{i}+(\alpha+7 \gamma) \sum_{j \in V(i)} q_{j}+6 \gamma\left\langle\prod_{j \in V(i)} \sigma_{j}\right\rangle
$$

For the leaves of the tree

$$
\frac{d}{d t} q_{i}=-q_{i}+q_{j} \tanh \beta J
$$


where $j$ is the neighbour of $i$. The factorization approximation consists in decoupling the third order correlations into

$$
\left\langle\prod_{j \in V(i)} \sigma_{j}\right\rangle=\prod_{j \in V(i)} q_{j}
$$

This approximation leads to the bulk behavior in the high temperature phase and in the vicinity of the transition. We start from a configuration of spins such as $q_{i}(0)=q_{j}(0)$ if the sites $i$ and $j$ belong to the same generation. Then, for $t>0, q_{i}(t)=q_{j}(t)$ if we work with the factorized dynamics. The factorized dynamics depends only on $n$ variables, one per generation, and is

$$
\begin{aligned}
\frac{d q_{n}}{d t} & =-q_{n}+3(\alpha+7 \gamma) q_{n-1}+6 \gamma q_{n-1}^{3} \\
\frac{d q_{i}}{d t} & =-q_{i}+(\alpha+7 \gamma)\left(2 q_{i-1}+q_{i+1}\right)+6 \gamma q_{i-1}^{2} q_{i+1} \\
\frac{d q_{1}}{d t} & =-q_{1}+q_{2} \tanh \beta J
\end{aligned}
$$

where $i$ runs from 2 to $n-1$. The equilibrium properties are calculated from the asymptotic values of the dynamics. We note that the factorized dynamics possesses a non-trivial fixed point for a finite size system, whereas the complete dynamics possesses only the Boltzman distribution as a fixed point. The asymptotics of the factorized dynamics is found to reproduce quite well the bulk properties of the tree. On figure 14, $q_{n}(+\infty)$ is plotted as a function of the inverse temperature. This curve is in agreement with the fact that the central spin exhibits a mean field like transition at $\beta_{c} \simeq 0.54$. We also plotted the asymptotic magnetization of the $m$ generations which are the closest to the central spin. It is clear that the predictions of the factorized dynamics are qualitatively wrong as soon as one goes out from the center. For instance, it is clear that the entire tree does not develop a transition at $\beta=\beta_{c}$. We note that, in equations (86), the transition temperature is determined entirely by the stability of the zero fixed point of the linear problem. Below $T_{c}$, the largest eigenvalue of the linear problem is positive, and negative above $T_{c}$. As in the Ginzburg-Landau theory, the nonlinear terms are responsible for the maximum bound on the dynamical variables in the low- temperature phase.

\section{Monte Carlo Dynamics}

\subsection{Relaxation of a Single kKink}

We begin by considering the case of a single kink and look for the relaxation of this excitation at low temperatures. Let's call $K$ the set of descendants of the kink. At time $t=0$, the configuration of kinks is such as $\sigma_{i}=-1$ is $i \in K$ and $\sigma_{i}=1$ if $i \notin K$. We follow the magnetization of the spins of $K$ as a function of time, for various size of $K$. The result is plotted on figure 15. The rapid relaxation at small times is attributed to the fact that the initial state is not thermalized; the thermalization occurs at small time scales compared to the collective processes of crossing the barrier. We define the typical relaxation time $\tau$ as the time-scale associated with the vanishing of the magnetization. 
In Figure 16 we have plotted the logarithm of this relaxation time, $\ln \tau$, as a function of the number of generations of $K\left(n_{K}\right)$. The points are approximately aligned, indicating that the Arrhenius law

$$
\tau \sim \tau_{0} \exp \left(\beta \lambda \frac{z-2}{2} n\right)
$$

is well-satisfied. The case $\lambda=1$ corresponds to the relaxation of a $n$-half-space tree where the ancestor is free. However, in our case, the ancestor is not free since the domain $K$ is connected to the remaining up spins. The barrier height is essentially the same as in the case $\lambda=1$, but the number of paths to reverse the magnetization is changed.

\subsection{The A.C. Susceptibility}

Experimentally, the a.c. nonlinear susceptibility has been a very useful probe of glassiness, 24] and we can apply the same techniques numerically to study the tree problem that we are discussing here. In parallel with what is done experimentally, a small a.c. external field $H(t)=h \sin \omega t$ is applied to the spin system, and we measure the magnetization response, expanded into its Fourier components

$$
M(\omega, t)=\sum_{k \geq 0} \theta_{k}^{\prime} \sin k \omega t+\theta_{k}^{\prime \prime} \cos k \omega t,
$$

which has only odd harmonics. In practice, we let the system relax for four periods and we take measurements only during the fifth one. The in-phase susceptibilities are related to the in-phase Fourier coefficients as [24]

$$
\begin{aligned}
\theta_{1}^{\prime} & =\chi_{1}^{\prime} h+\frac{3}{4} \chi_{3}^{\prime} h^{3}+\frac{5}{8} \chi_{5}^{\prime} h^{5}+\frac{35}{64} \chi_{7}^{\prime} h^{7}+\ldots \\
\theta_{3}^{\prime} & =\frac{1}{4} \chi_{3}^{\prime} h^{3}+\frac{5}{16} \chi_{5}^{\prime} h^{5}+\frac{21}{64} \chi_{7}^{\prime} h^{7}+\ldots \\
\theta_{5}^{\prime} & =\frac{1}{16} \chi_{5}^{\prime} h^{5}+\frac{7}{64} \chi_{7}^{\prime} h^{7}+\ldots \\
\theta_{7}^{\prime} & =\frac{1}{64} \chi_{7}^{\prime} h^{7}+\ldots
\end{aligned}
$$

Since all the nonlinear susceptibilities are divergent at the critical temperature of a spin glass, 24] we must include all the measurable higher order harmonics in order to properly include their influence on the lower ones. 24].

The susceptibilities $\chi_{1}^{\prime}$ and $\chi_{3}^{\prime}$ are plotted as a function of temperature in Figures 17 and 18 respectively for a number of frequencies. Both $\chi_{1}^{\prime}$ and $\chi_{3}^{\prime}$ display maxima, where that of the latter is more pronounced; in a "real" spin glass in the thermodynamic limit one would expect $\chi_{1}^{\prime}$ to have a maximum, and $\chi_{3}^{\prime}$ to diverge. We note that $\chi_{3}^{\prime}$ is negative near its maximum, which agrees with experiments on spin glasses [24]. The frequency dependence of the position of the maximum in $\chi_{1}^{\prime}$ and $\chi_{3}^{\prime}$ is also consistent with experiment, 24] since the temperature for which $\chi_{1}^{\prime}$ and $\chi_{3}^{\prime}$ are maximum increases with frequency. Thus this study strongly suggests that the Ising model on a finite Cayley tree exhibits glassy-like behavior at low temperatures; we shall be more specific when we analyze the Edwards-Anderson susceptibility of this model. 


\subsection{Autocorrelation Functions and Ageing}

A good test used to determine the presence of glassiness in a given model involves the computation of the autocorrelation functions [25] to determine whether they exhibit ageing. The autocorrelation functions are

$$
C\left(t, t_{w}\right)=\frac{1}{N} \sum_{i=1}^{N}\left\langle\sigma_{i}\left(t+t_{w}\right) \sigma_{i}\left(t_{w}\right)\right\rangle-\left\langle\sigma_{i}\left(t+t_{w}\right)\right\rangle\left\langle\sigma_{i}\left(t_{w}\right)\right\rangle,
$$

where the sample is rapidly quenched below $T_{g}$ from a disordered high temperature state. The Monte Carlo dynamics runs from times $t=0$ to $t=t_{w}$; the autocorrelations are measured at $t=t_{w}$ where an average is taken over the initial configurations.

The autocorrelations in the high-temperature phase are plotted on figure 19. They decrease rapidly with the time $t$ and are independent of the waiting time, as expected; this is simply a check of our code. Figure 20 represents the autocorrelation functions below $T_{g}$. The aucorrrelations increase with increasing $t_{w}$, indicating the presence of ageing. Such behavior has been observed in a wide class of glassy models, including the fully frustated hypercubic model [25].

\section{The Edwards-Anderson Order Parameter and Susceptibility}

The recursive structure of the Cayley tree permits us to compute the Edwards-Anderson order parameter and susceptibility at all temperatures. This calculation has already been done for the $\pm J$ model with uncorrelated boundary conditions in [10] [14]. These authors found an Almeida-Thouless line for their spin glass model, and we wish to use the same technique to analyse the model discussed here. In order to compute the Edwards-Anderson order parameter and susceptibility, we consider one tree plus one replica, with an intereplica coupling $R$. If $\{\sigma\}$ and $\left\{\sigma^{\prime}\right\}$ are the spin configurations of the two replicas, the Hamiltonian reads

$$
H=-J \sum_{\langle i, j\rangle}\left(\sigma_{i} \sigma_{j}+\tilde{\sigma}_{i} \tilde{\sigma}_{j}\right)-R \sum_{i=1}^{N} \sigma_{i} \tilde{\sigma}_{i}+H \sum_{i=1}^{N}\left(\sigma_{i}+\tilde{\sigma}_{i}\right) .
$$

As per usual with free boundary trees, we have to distinguish between the properties of the central spin and the whole tree; in what follows we discuss both cases below.

\subsection{The whole tree}

The partition function can be calculated by the construction of figure 2, even in the presence of the intereplica coupling. We take derivatives of the partition function with respect to the intereplica coupling, $R$, resulting in the Edwards-Anderson order parameter

$$
q_{E A}=\frac{1}{N}\left\langle\sum_{i=1}^{N} \sigma_{i} \tilde{\sigma}_{i}\right\rangle=\frac{1}{N} \frac{\partial}{(\partial \beta R)} \ln Z(R=0)
$$


and the Edwards-Anderson susceptibility:

$$
\chi_{E . A .}=\frac{1}{N}\left(\left\langle\left(\sum_{i=1}^{N} \sigma_{i} \tilde{\sigma}_{i}\right)^{2}\right\rangle-\left\langle\sum_{i=1}^{N} \sigma_{i} \tilde{\sigma}_{i}\right\rangle^{2}\right)=\frac{1}{N} \frac{\partial}{\partial(\beta R)^{2}} \ln Z(R=0) .
$$

We now let $Z_{\sigma \sigma^{\prime}}^{(n)}$ be the conditional partition function of a $n$-half space tree with respect to the ancestor's spins $\sigma$ and $\sigma^{\prime}$. It is clear that

$$
\begin{aligned}
& Z_{++}^{(n+1)}=e^{\beta R} e^{2 \beta H}\left(e^{2 \beta J} Z_{++}^{(n)}+Z_{+-}^{(n)}+Z_{-+}^{(n)}+e^{-2 \beta J} Z_{--}^{(n)}\right)^{z-1} \\
& Z_{+-}^{(n+1)}=e^{-\beta R}\left(Z_{++}^{(n)}+e^{2 \beta J} Z_{+-}^{(n)}+e^{-2 \beta J} Z_{-+}^{(n)}+Z_{--}^{(n)}\right)^{z-1} \\
& Z_{-+}^{(n+1)}=e^{-\beta R}\left(Z_{++}^{(n)}+e^{-2 \beta J} Z_{+-}^{(n)}+e^{2 \beta J} Z_{-+}^{(n)}+Z_{--}^{(n)}\right)^{z-1} \\
& Z_{--}^{(n+1)}=e^{\beta R} e^{2 \beta H}\left(e^{-2 \beta J} Z_{++}^{(n)}+Z_{+-}^{(n)}+Z_{-+}^{(n)}+e^{2 \beta J} Z_{--}^{(n)}\right)^{z-1} .
\end{aligned}
$$

The initial conditions of the recursion are $Z_{++}^{0}=e^{\beta R} e^{2 \beta H}, Z_{+-}^{0}=Z_{-+}^{0}=e^{-\beta R}$ and $Z_{--}^{0}=$ $e^{\beta R} e^{-2 \beta H}$. For all $n$, we can show that $Z_{+-}^{(n)}=Z_{-+}^{(n)}$ and we then define $Z_{1}^{(n)} \equiv Z_{++}^{(n)}, Z_{0}^{(n)} \equiv Z_{+-}^{(n)}=$ $Z_{-+}^{(n)}$ and $Z_{-1}^{n} \equiv Z_{--}^{(n)}$. Using this notation, the recursion relations become

$$
\begin{gathered}
Z_{1}^{(n+1)}=e^{\beta(R+2 H)}\left(e^{2 \beta J} Z_{1}^{(n)}+2 Z_{0}^{(n)}+e^{-2 \beta J} Z_{-1}^{(n)}\right)^{z-1} \\
Z_{0}^{(n+1)}=e^{-\beta R}\left(Z_{1}^{(n)}+2 \cosh \beta J Z_{0}^{(n)}+Z_{-1}^{(n)}\right)^{z-1} \\
Z_{-1}^{(n+1)}=e^{\beta(R-2 H)}\left(e^{-2 \beta J} Z_{1}^{(n)}+2 Z_{0}^{(n)}+e^{2 \beta J} Z_{-1}^{(n)}\right)^{z-1} .
\end{gathered}
$$

which can be used to obtain $q_{E A}$ and $\chi_{E A}$ in a straight-forward fashion . $q_{E A}$ and $\chi_{E A}$ are plotted in figures 21 and 22 respectively for $n=10$ and $n=80$. In both cases, the curves corresponding to these two system sizes are similar, even though the number of sites is small for $n=10$ (2047 sites) and macroscopic for $n=80$ (4.02 moles of sites). We believe that this behavior is related to the very slow variation of the glass temperature $T_{g}$ with the system size. In figure 22 we see clearly that

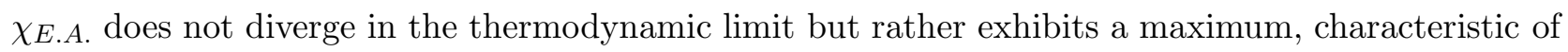
a finite size effect, even in the macroscopic regime.

\subsection{The central spin}

Following the authors of [10] [14], we note that

$$
\begin{aligned}
& Z^{(n)}=Z_{1}^{(n)}+2 Z_{0}^{(n)}+Z_{-1}^{(n)} \\
& Q^{(n)}=\frac{1}{Z^{(n)}}\left(Z_{1}^{(n)}-2 Z_{0}^{(n)}+Z_{-1}^{(n)}\right) .
\end{aligned}
$$

The Edwards-Anderson order parameter of the central spin is $\tilde{q}_{E . A .}=Q^{(n)}$, and the EdwardsAnderson susceptibility is

$$
\tilde{\chi}_{E . A .}^{(n)}=\frac{\partial \tilde{q}_{E . A .}}{(\partial \beta R)}(R=0)
$$


where the tilde symbol denotes quantities with respect to the central spin. The "central spin"

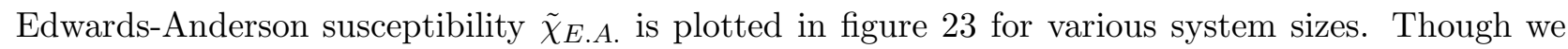
have seen that finite-size effects are negligeable for the whole tree, they become crucial for the central spin. More specifically, the maximum values of the Edwards Anderson susceptibility of the central spin does not increase (see figure 24) as a function of coordination $z$. As the system size becomes macroscopic, the glass temperature $T_{g}$ depends more on the coordination than on the number of generation; in particular, it increases with increasing coordination consistent with equation (43).

\subsection{Central spin in a magnetic field}

In the Sherrington-Kirkpatrick model, glassy behavior persists even in the presence of a magnetic field; the Edwards-Anderson susceptibility diverges with an exponent $\gamma=1$ through the AlmeidaThouless line. We have studied the behavior of the Ising n.n. ferromagnet (fm) on a Cayley tree ferromagnetic model with free boundary conditions with an applied magnetic field. Specifically we studied the variation of the Edwards-Anderson susceptibility in a field as a function of system size. As displayed in figure 25, the maximum of the Edwards-Anderson susceptibility as a function of temperature depends on the size. In figure 26, we showthe locus of the maxima of $\tilde{\chi}_{E . A \text {. for different }}$ magnetic fields as a function of temperature. The Edwards-Anderson susceptibility decreases strongly with increasing magnetic field, indicating the vanishing of glassy behavior consistent with expectation.

\section{Lee and Yang zeros}

The partition function of the Ising fm on the Cayley tree in the absence of a magnetic field is equal to that of the linear chain, so that the Lee and Yang zeros in the plane of exp $2 \beta$ have the identical structure in both models. Clearly this will not be the case for finite applied field. We can introduce a complex magnetic field and determine the zeros of the partition function in the complex plane of exp $2 \beta h$. Since we do not impose a uniform magnetic field, but rather one only on the leaves of the tree, we can compute the zeros using the recursion relations (5), which involve only second order equations. Since the magnetic field is non-uniform, the underlying assumptions of the circle theorem [27] are not fulfilled. The zeros are not exactly on the unit circle but areb in its vicinity. More precisely, numerical work indicates that the zeros approach the unit circle with increasing generation. The zeros are plotted on figure 27 in the cases $\beta<\beta_{c}, \beta=\beta_{c}$ and $\beta>\beta_{c}$. In figure 28, we exhibit the ratio of the density of zeros in the vicinity of $\exp 2 \beta h=1$ over the averaged density on the circle. The ratio is zero for temperatures larger than the bulk transition temperature, increases to a small positive value below the bulk transition temperature, and eventually becomes larger than unity below a certain temperature. In the case of a Weiss model, the density of zeros $g(\theta)$ is given by 28

$$
g(\theta)=\frac{1}{2 \pi}(1-2 r \cos \varphi),
$$


where

$$
\begin{aligned}
r & =\sqrt{4+r^{2}-4 r \cos \varphi} \exp \left(-2 \frac{T_{c}}{T}(1-r \cos \varphi)\right) \\
\theta & =-2 \frac{T_{c}}{T} r \sin \varphi+\varphi+\arctan \left(\frac{r \sin \varphi}{2-r \sin \varphi}\right) .
\end{aligned}
$$

The ratio of the density of zeros at the point $\exp 2 \beta h=1$ over the average density of zeros can be determined numerically from the sysetem of equations above, and is plotted on the inset of figure (28), and is always less than unity. By contrast on the tree, the density of zeros in the vicinity of the real axis is thus abnormally high below a certain temperature, which we identify with the temperature scale $T_{g}$.

\section{Discussion}

In summary, we have studied the static and the dynamical properties of the nearest-neighbor Ising model on a Cayley tree. At zero field, we find that this system displays glassy behavior below a size-dependent temperature that scales inversely with the logarithm of the number of generations; thus its glassy behavior persists for a finite but macroscopic number of sites. Because the ratio of the number of surface to bulk sites, $\frac{N_{s}}{N}$, and the strength of the external field, $h$, play a key role in the physical behavior of the resident spin system, the different thermodynamic limits associated with the values of $\frac{N_{s}}{N}$ and $h$ are characterized; the cross-over temperature, $T_{g}$, is associated with fixed $\frac{N_{s}}{N}$ in the limit of vanishing applied field. Physically well-defined large domains of flipped spins develop at $T_{g}$; at this temperature the probability of nested spin clusters is small. The largest energy barriers associated with overturning these domains is determined to scale logarithmically with the number of sites at zero temperature, a result that should be valid at finite, low temperatures if overlap between spin clusters does not occur. A dynamical study indicates the appearance of metastable states and long relaxation times at low temperatures. The autocorrelations are computed after a waiting time using Monte Carlo dynamics; they exhibit ageing for $T<T_{g}$. The temperature variations of the coefficients of $\chi_{1}^{\prime}$ and $\chi_{3}^{\prime}$ are also determined and they agree with the existence of finite-size glassiness. Finally the Edwards-Anderson susceptibility of the entire tree displays a maximum (but no divergence) that evolves slowly with increasing system size; that of the central spin has much more marked size-dependence.

We have thus performed a detailed characterization of the low-temperature phase of a shortrange periodic spin model resident on a Cayley tree. In this particular case, we have found that it displays finite-size glassy behavior that remains for a macroscopic number of sites; perhaps it is best to characterize this low-temperature phase as a very viscous spin liquid. We note that neither

intrinsic disorder nor frustration exist due to the initial Ising Hamiltonian; the possibility of many low-temperature "cluster" states separated by very high energy barriers is a direct consequence of 
the unusual topology of the Cayley tree. In many ways we hope that this is a warm-up exercise towards the study of spin models on more complicated non-Euclidean lattices, e.g. on a constant triangulation associated with a surface of negative curvature, where the intrinsic geometry of the host may lead to the possibility of glassiness in the absence of both disorder and frustration.

\section{Appendix A}

We propose another derivation of the magnetization distribution for the tree. Let $Z(\beta, h)$ the partition function of the spin system in the presence of an external field. Then,

$$
P(M)=\frac{1}{Z(\beta, h)} \sum_{\{\sigma\}} \delta\left(M-\sum_{i=1}^{N} \sigma_{i}\right) \exp \left(\beta\left(J \sum_{\langle i, j\rangle} \sigma_{i} \sigma_{j}+h \sum_{i=1}^{N} \sigma_{i}\right)\right) .
$$

Using the Fourier representation of the delta function

$$
\delta\left(M-\sum_{i=1}^{N} \sigma_{i}\right)=\frac{1}{2 \pi} \int_{0}^{2 \pi} d \lambda e^{i \lambda M} e^{-i \lambda \sum_{i=1}^{N} \sigma_{i}},
$$

we obtain

$$
P(M)=\frac{1}{2 \pi} \int_{0}^{2 \pi} d \lambda e^{i \lambda M} \frac{Z(\beta, h-i \lambda / \beta)}{Z(\beta, h)},
$$

where we have used the analytic continuation of the partition function for complex magnetic fields. This method is usefull provided one knows how to calculte the partition function, which is certainly feasible on a tree. We proceed by decimation, starting from the border of the $n$-half-space-tree. For future purpose, we note $Z_{n}\left(\beta, h, h_{n}\right)$ the partition function of a $n$-half-space-tree with a magnetic field $h$ acting on the spins of the generations 0 to $n-1$, and $h_{n}$ on the spins of the generation $n$. Then,

$$
Z_{n}\left(\beta, h, h_{n}\right)=\left(4\left(\cosh ^{2}(\beta J)+\sinh ^{2}\left(\beta h_{n}\right)\right)^{\frac{(z-1)^{n-1}}{2}} Z_{n-1}\left(\beta, h, h+T . h_{n}\right),\right.
$$

where the transformation of the magnetic field reads

$$
T . h=\frac{z-1}{2 \beta} \ln \frac{\cosh \beta(J+h)}{\cosh \beta(J-h)} .
$$

The last term of the recursion corresponds to the partition function of the ancestor, which is simply

$$
Z_{1}(\beta, h)=2 \cosh \left(\beta T^{n} \cdot h\right)
$$

It is straighforward to compute the partition function using these relations and to perform the Fourier transform (112) in order to obtain the probability distribution of the magnetization. 


\section{Appendix B}

In this appendix we give the value of the energy barrier $E\left(T_{n, z}\right)$ for a half-space-tree $T_{n, z}$ with $\mathrm{n}$ generations and a coordination number of $z$ for all sites except the root (coordination $z-1$ ) and the leaves (coordination 1). We also give the energy barrier $E\left(T_{n, z}^{*}\right)$ for a complete tree $T_{n, z}^{*}$ with $\mathrm{n}$ generations and a coordination number of $z$ for all sites except the leaves. The derivation of the formula is due to A. Sebő and M. Preissmann, and is published in extenso in 29. Note that the same problem arises in the VLSI circuit conception! Generically the problem of finding the lowest energy barrier is NP-complete, but the sub-problem of finding the lowest energy barrier of a tree is polynomial, and an explicit algorithm is given hereafter. The value of the energy barriers are given by :

$$
\begin{array}{r}
c\left(T_{n, z}\right)=\left\lceil\frac{n(z-2)}{2}\right\rceil+1 \quad(n, z \geq 3) \\
c\left(T_{n, z}^{*}\right)=\left\lceil\frac{(n-1)(z-2)}{2}\right\rceil+\left\lceil\frac{z-2}{2}\right\rceil+1 \quad(n, z \geq 3)
\end{array}
$$

In the above formula $\lceil x\rceil$ denotes the lowest of the integers greater than $x$. The demonstration of these formula is constructive. Firstly a lower bound for $E$ is given. Then an algorithm is described which produces a labeling of the sites. Flipping the spins in the order of this labeling gives an energy barrier exactly equal to the lower bound. The algorithm is recursive. It tries to produce an optimal labeling of the sites where the root is labeled before the configuration of highest energy is reached. We call strong labeling such a labeling. This extra constraint is useful when one applies $z$ times the algorithm on a $T_{n, z}$ to compute $E\left(T_{n+1, z}\right)$, or when one applies the algorithm to $T_{n, z}$ and to $T_{n-1, z}$ to compute $E\left(T_{n+1, z}^{*}\right)$. A strong labeling does not exist when $z$ and $n$ are both odd as shown in [29]. Let us now consider on the case of $T_{n, z}$. Formula 116 means that

- when $z$ is even the increment in energy when one goes from $T_{n, z}$ to $T_{n+1, z}$ is constant and equal to $\frac{z}{2}-1$

- when $z$ is odd the increment in energy when one goes from $T_{n, z}$ to $T_{n+1, z}$ is alternatively $\frac{z-1}{2}-1$ and $\frac{z-1}{2}$

We give now the algorithm in the case of even $z$. The case of odd $z$ is a slightly more complicated, but in the same spirit. Consider $T_{n, z}$ as being made of $z-1$ copies of $T_{n-1, z}$ all of them connected to the site 0 . Each spin is identified by two numbers $k, i$ with $0 \leq k<z-1$ and $0 \leq i<N_{n-1}$ $\left(N_{n}\right.$ is the number of sites of $T_{n, z}$ ). Let us note $\pi$ a strong labeling of $T_{n-1, z}$, and $n_{0}$ the root of the $\left(\frac{z-2}{2}-1\right)$ th copy of $T_{n-1, z}$. The following labeling is a strong labeling of $T_{n, z}$ :

1. $(0, \pi(0)),(0, \pi(1)), \ldots,\left(0, \pi\left(N_{n-1}\right)\right)$

2. ... 
3. $\left(\frac{z-2}{2}-1, \pi\left(N_{n}\right)\right),\left(\frac{z-2}{2}-1, \pi\left(N_{n}-1\right)\right), \ldots,\left(\frac{z-2}{2}-1, \pi\left(n_{0}\right)\right)$

4. 0

5. $\left(\frac{z-2}{2}-1, \pi\left(n_{0}-1\right)\right),\left(\frac{z-2}{2}-1, \pi\left(n_{0}-2\right)\right), \ldots,\left(\frac{z-2}{2}-1, \pi(0)\right)$

6. $\left(\frac{z-2}{2}, \pi(0)\right),\left(\frac{z-2}{2}, \pi(1)\right), \ldots,\left(\frac{z-2}{2}, \pi\left(N_{n-1}\right)\right)$

7. ...

8. $(z-2, \pi(0)),(z-2, \pi(1)), \cdots,\left(z-2, \pi\left(N_{n-1}\right)\right)$

Note that in step 1, 2, 7, and 8 any admissible permutation can be used instead of the strong labeling

$\pi$. It is shown in [29] that the above labeling is indeed an optimal labeling and it can be used to implement a recursive algorithm to find a path between the two ferromagnetic states. 


\section{References}

[1] For a pedagogical review of recursive structures see M.F. Thorpe in Excitations in Disordered Solids (NATO Advanced Study Institute Series B, ed. M.F. Thorpe (New York: Plenum, 1982), pp 85-107.

[2] C. Domb, Adv. Phys., 9, 145 (1960).

[3] e.g. R.J. Baxter, Exactly Solved Models in Statistical Mechanics, (Academic Press, London, 1982), Chapter 4.

[4] P.D. Gujrati, Phys. Rev. Lett., 74, 809 (1995).

[5] D.J. Thouless, P.W. Anderson and R.G. Palmer, Phil.Mag., 35, 593 (1977).

[6] D. Sherrington and S. Kirkpatrick, Phys. Rev. Lett., 35, 1792 (1975).

[7] D. Bowman and K. Levin, Phys. Rev. B, 25, 3438 (1982).

[8] D.J. Thouless, Phys. Rev. Lett., 56, 1082, (1986).

[9] S. Katsura, Prog. Theo. Phys., 87, 139 (1986).

[10] J.T. Chayes, L. Chayes, J.P. Sethna and D.J. Thouless, Comm. Math. Phys., 106, 41 (1986).

[11] P. Mottishaw, Europhys. Lett., 4, 333 (1987).

[12] Pik-Uin Lai and Y.Y. Goldschmidt, J. Phys. A, 22, 399 (1989).

[13] J.M. Carlson, J.T. Chayes, L. Chayes, J.P. Sethna and D.J. Thouless, Europhys. Lett., 5, 355 (1988); J. Stat. Phys., 61, 987 (1990).

[14] J.M. Carlson, J.T. Chayes, J.P. Sethna and D.J. Thouless, J. Stat. Phys., 61, 1069 (1990).

[15] Y.Y. Goldschmidt, Phys. Rev. B, 43. 8148 (1991).

[16] P.J. Gujrati, Phys. Rev. Lett., 53, 2453 (1984); J. Chem. Phys., 98, 1613 (1993).

[17] R.B. Stinchcombe, J. Phys. C, 6, L1 (1973).

[18] R. Rammal, J. Physique, 46, 1837 (1985); R. Rammal and A. Benoit, J. Physique Lett., 46, L-667 (1985); Phys. Rev. Lett., 55, 649 (1985).

[19] T.P. Eggarter, Phys. Rev. B, 9, 2989 (1974).

[20] M. Matsuda, Prog. Theor. Phys., 51, 1053 (1974). 
[21] J. von Heimburg and H. Thomas, J. Phys. C, 7, 3433 (1974).

[22] E. Muller-Hartmann and J. Zittartz, Phys. Rev. Lett., 33, 893 (1974).

[23] R.J. Glauber, Jour. Math. Phys. $\underline{4}, 294$ (1963).

[24] L.P. Lévy, Phys. Rev. B $\underline{38}, 4963$ (1988).

[25] E. Marinari, G. Parisi and F. Ritort, preprint cond-mat/9410089.

[26] M. Kurata, R. Kikuchi and T. Watari, J. Chem Phys, 21, 434 (1953).

[27] C.N. Yang, T.D. Lee, Phys. Rev. $\underline{87}, 404$ (1952), T.D. Lee, C.N. Yang, Phys. Rev. $\underline{87}, 410$ (1952). See also K. Huang, Statistical Mechanics, Wiley, N.Y. (1963).

[28] R. Rammal, thèse d'état (1981).

[29] J. C. Anglès d'Auriac, M. Preissmann and A. Sebő submitted to Journal of Mathematics and Combinatorics (1995). 


\section{Figure captions}

Figure 1:

(a) A finite Cayley tree and (b) a section of a Bethe lattice with coordination $z=3$.

Figure 2:

A recursive construction of half-space trees.

Figure 3:

$h_{i+1}^{i n d}\left(h_{i}\right)$ where $h_{i}$ is the total magnetic field at generation $i$, and $h_{i+1}^{i n d}$ is the iterated field of equation (5). The three curves correspond to (a) $\beta<\beta_{c}$, (b) $\beta=\beta_{c}$ and (c) $\beta>\beta_{c}$.

Figure 4:

The density probability of the magnetization on a half-space tree where $n=10, z=3$, the ancestral spin is fixed, and the inverse temperature is $\beta=3$.

Figure 5:

The conditional magnetization distribution in the presence of a magnetic field, the field being parallel to the central spin. The magnetization distribution is plotted for a $z=3$ tree, $n=10$ generations, and for magnetic fields $H=0,0.001,0.002$. The magnetization distribution evolves towards a gaussian shape as the magnetic field increases.

Figure 6:

A typical configuration of the tree with $n=8$ and $\beta=1.5$. The vertices with no dots represent up spins and the dots represent flipped spins. Each kink gives rise to a well-defined domain of flipped spins.

\section{Figure 7:}

Eigenvalues of the Glauber matrix as a function of the inverse temperature for an open six-site Ising chain (64 states). The evolution of the energy levels as a function of $\beta$ is monotonous, and the eigenvalues are free to cross each other.

Figure 8:

Spectrum of the Glauber matrix at a given temperature for a ring of ten sites. The inverse temperature is $\beta=3$. No gap is present. 


\section{Figure 9:}

Spectrum of the Glauber matrix at a given temperature for a 3x3 Ising model with open boundary conditions. The inverse temperature is $\beta=3$. Some degeneracies appear in the spectrum, and the spectrum is symmetric with respect to the $\lambda=-N / 2$ line.

Figure 10:

Eigenvalues of the Glauber matrix as a function of the inverse temperature for a $3 \times 2$ Ising cluster. The number of sites is 6 , leading to 64 states. The evolution of the eigenvalues as a function of $\beta$ is non monotonous, and avoided crossings are visible.

\section{Figure 11:}

Eigenvalues of the Glauber matrix as a function of the inverse temperature for a $n=1, z=3$ Cayley tree. The number of sites is four, leading to 16 states. The evolution of the energy levels as a function of $\beta$ is not monotonous for certain levels, indicating level repulsion.

Figure 12:

Spectrum of the Glauber matrix at a given temperature for $z=3$ Cayley tree with 2 generations. The inverse temperature is $\beta=2$. The spectrum is symmetric with respect to the $\lambda=-N / 2$ line. The clusters collapse at integer values.

\section{Figure 13:}

Evolution of the magnetization of the 16 pure states of the tree with one generation. The inverse temperature is $\beta=3$. Metastable states appear to be present even for such a small size. The insert represents the same curve at short time scales, which shows the transient regime from the natural basis of pure states to the metastable states.

\section{Figure 14:}

Expectation value of the central spin in the simplified dynamics, as a function of the inverse temperature. The transition temperature is in agreement with the value $\beta_{C} \simeq 0.54$. The shape of the curve near the transition is in agreement with the existence of a mean field like transition for the central spin. We also plotted the expectation value of the magnetization of the $m$ closest to the origin slices of spins, in the factorisation approximation, for $m=3,4,5,6,7,8,9,10$.

Figure 15:

Magnetization relaxation of a single kink. Initially, all the spins of a $z=3, n=10$ tree are up, and one creates a kink. We call $n_{0}$ the number of generations involved in the kink. If $n_{0}=1$, the kink has only one spin, if $n_{0}=2$, the kink has 7 spins, etc. We follow the magnetization of this domain as a function of time. The unit time is one Monte Carlo Step (M.C.S.). One M.C.S. corresponds to repeating $N$ times the process which consists in choosing one spin at random among the $N$ sites, and changing or not its direction, according to the Boltzman distribution. The curves are averaged over 50 different Monte Carlo runs of the dynamics. 
Figure 16:

Logarithm of the relaxation time as a function of the number of generations in the kink. The relaxation time is defined from the cancelation of magnetization on figure 15. The points are approximately aligned, which is in agreement with the Arrhenius law of equation (87).

Figure 17:

$\chi_{1}^{\prime}$ susceptibility on the Cayley tree. The tree has 10 generations. The amplitude of the magnetic field is 0.1. The curves are averaged over 50 initial configurations of spins, generated at equilibrium. One has to find a compromise between the amplitude of the magnetic field and the number of configurations to be averaged over, to have a good signal/noise ratio. The curves correspond to a period of the magnetic field equal to 500 M.C.S., 1000 M.C.S. and 1500 M.C.S..

Figure 18:

$\chi_{3}^{\prime}$ susceptibility on the Cayley tree. The tree has 10 generations. The amplitude of the magnetic field is 0.1 . The curves are averaged over 50 initial configurations of spins, generated at equilibrium. The curves correspond to a period of the magnetic field equal to 500 M.C.S., 1000 M.C.S. and 1500 M.C.S.. 
Figure 19:

Spin autocorrelation functions of the $z=3$ Cayley tree above $T_{g}$. The unit time is one M.C.S., the inverse temperature is $\beta=0.5$, and the averages are taken over 100 random initial configurations. The autocorrelation functions decrease rapidly with $t$ and are independant on $t_{w}$.

Figure 20:

Spin autocorrelation functions of the $z=3$ Cayley tree below $T_{g}$. The unit time is one M.C.S., the inverse temperature is $\beta=2$, and the averages are taken over 100 random initial configurations. The autocorrelation functions depend on the waiting time, and increase with the waiting time, which is the signature of glassiness.

Figure 21:

Edwards-Anderson order parameter for the whole spin system. The coordination is $z=3$. The Edwards-Anderson order parameter is plotted as a function of temperature for $n=10$ (2047 spins) and $n=80$ (4.02 moles of spins). The two curves nearly coincide.

Figure 22:

Edwards-Anderson susceptibility for the whole spin system. The coordination is $z=3$. The EdwardsAnderson susceptibility is plotted as a function of temperature for $n=10$ (2047 spins) and $n=80$ (4.02 moles of spins). The two curves nearly coincide.

Figure 23:

Edwards-Anderson susceptibility for the central spin as a function of temperature for $n=$ $5,10,15,20,25,30,35,40$ generations. The coordination is $z=3$.

Figure 24:

Edwards-Anderson susceptibility for the central spin as a function of temperature for $z=3,4,5$. The number of generations is $n=20$.

Figure 25:

Edwards-Anderson susceptibility of the central spin in a magnetic field as a function of temperature, for different sizes. The number of generations is $\mathrm{n}=2,3,4,5,6,7,8,9,10,30$. The magnetic field is $H=0.05$ 
Figure 26:

Maxima of $\chi_{E A}$ as a function of temperature for different values of the magnetic field. The system size is $n=10$ generations. The magnetic fields are $H=0.025, H=0.05, H=0.1, H=0.15, H=0.2$.

\section{Figure 27:}

Zeros of Lee and Yang at different temperatures. The radial coordinate is rescaled in order to allow the superposition of different maps of zeros. The external map represents the zeros for 12 generations, $z=3$ and $\beta=0.3$. In this case, the zeros are localized on well defined areas of the complex plane, far from the real axis. The intermediate map represents the case $\beta=\beta_{c} \simeq 0.54$. The zeros begin to fill the circle, with areas of zero density, espacially at the intersection of the real axis with the unit circle. The inner map represent the zeros at low temperature, $\beta=2$. In this case, the circle is filled with zeros. An analysis of the density of zeros reveals an annormally high density in the vicinity of the point $h=0$.

Figure 28:

Ratio of the density of zeros in the vicinity of the point exp $2 \beta h=0$ over the average density of zeros in the case of the $z=3$ tree with 17 generations. The inset represents the same quantity for a Weiss model with $T_{c}=1$. In the case of the tree, the ration is greater than one below a certain temperature, whereas it is always less than unity for the Weiss model. 